\title{
Radio Resource Allocation for Uplink OFDMA Systems with Finite Symbol Alphabet Inputs
}

\author{
Mohammed Al-Imari, Pei Xiao, Muhammad Ali Imran, and Rahim Tafazolli
}

\begin{abstract}
In this paper, we consider the radio resource allocation problem for uplink OFDMA system. The existing algorithms have been derived under the assumption of Gaussian inputs due to its closed-form expression of mutual information. For the sake of practicality, we consider the system with Finite Symbol Alphabet (FSA) inputs, and solve the problem by capitalizing on the recently revealed relationship between mutual information and Minimum Mean-Square Error (MMSE). We first relax the problem to formulate it as a convex optimization problem, then we derive the optimal solution via decomposition methods. The optimal solution serves as an upper bound on the system performance. Due to the complexity of the optimal solution, a low-complexity suboptimal algorithm is proposed. Numerical results show that the presented suboptimal algorithm can achieve performance very close to the optimal solution and outperforms the existing suboptimal algorithms. Furthermore, using our proposed algorithm, significant power saving can be achieved in comparison to the case when Gaussian input is assumed.
\end{abstract}

Index Terms-Radio resource allocation, OFDMA, uplink, finite symbol alphabet.

\section{INTRODUCTION}

Orthogonal Frequency Division Multiple Access (OFDMA) is a key technology in existing and upcoming mobile communication systems, including 3GPP-LTE and WiMAX. The radio resource allocation in the OFDMA context has attracted considerable attention due to its importance in optimizing the OFDMA system performance. The radio resource allocation algorithms exploit the frequency and multiuser diversities to optimize the sum-rate of the system. In uplink, each user has individual power constraint, which makes the optimization problem more challenging. In the literature, the Subcarrier and Power Allocation (SPA) algorithms are based on the assumption of Gaussian signalling as the channel input [1]. This assumption is motivated by the closed-form expression of mutual information under the Gaussian input, which simplifies the problem definition. However, in practical systems, Finite Symbol Alphabet (FSA) are employed (e.g., M-QAM). The Signal to Noise Ratio (SNR) gap has been used to model the difference between the capacity given the Gaussian input and mutual information under FSA constraint in [2]. The main issue with the capacity of Gaussian inputs is that it is unbounded in the SNR. On the other hand, the mutual information given the FSA input is bounded by $\log _{2}|\mathcal{X}|$, where $\mathcal{X}$ denotes the constellation alphabet. Thus, the achievable rate based on the Gaussian assumption is not valid for practical systems. Furthermore, in [3], the advantage of explicitly considering FSA for single-user OFDM system is demonstrated. It has

The authors are with the Centre for Communication Systems Research, University of Surrey, Guildford, Surrey, GU2 7XH, UK (Email:\{m.al-imari, p.xiao, m.imran, r.tafazolli\}@surrey.ac.uk). been shown that unlike the water-filling solution for Gaussian inputs, for FSA inputs more power has to be allocated to the weak channels [3].

In this paper, we consider the joint SPA problem in uplink OFDMA system with FSA input. The objective is to maximize users' weighted sum mutual information. The SPA problem is complicated in the uplink OFDMA due to the individual power constraints and the discrete nature of subcarrier allocation. We approach this problem by first relaxing the integer allocation constraint and allowing more than one user to share the subcarrier. Then, we use the decomposition method to solve the relaxed problem by decoupling the main problem into two subproblems. To the best of our knowledge, the optimal SPA for multiuser uplink with FSA inputs has not been investigated in the literature. Although sharing the subcarriers may not be practical, solving the relaxed problem provides an upper bound on the system performance and can be used to evaluate the performance of the suboptimal algorithms. We also propose a low-complexity suboptimal algorithm that takes into consideration the integer allocation. In our solution, we exploit the recently revealed fundamental relationship between mutual information and Minimum Mean-Square Error (MMSE) [4]. The developed optimal solution and the proposed algorithm are valid for any input distribution, including Gaussian. It will be shown that our proposed suboptimal algorithm achieves performance that is very close to the optimal one and outperforms the existing suboptimal algorithms. It is also revealed that optimizing the power allocation based on FSA inputs saves the total transmitted power comparing to the case with the Gaussian assumption.

\section{SYSTEM MODEL}

Here, we consider the SPA problem for uplink OFDMA systems. The objective is to allocate the available subcarriers and power to maximize the weighted sum mutual information in the system. A single-cell OFDMA system is considered with a set of users $\mathcal{K}=\{1, \cdots, K\}$ transmitting to the same base station. The total frequency band is divided into a set of subcarriers $\mathcal{N}=\{1, \cdots, N\}$. The users are subject to individual maximum power constraints $\left(P_{k}\right)$ such that: $\sum_{n \in \mathcal{N}} p_{k, n} \leq P_{k}$, where $p_{k, n}$ is the transmission power of the $k$ th user on subcarrier $n$. The transmitted symbols are drawn from FSA. Channel State Information (CSI) is assumed to be available at the base station. The optimization problem for weighted sum mutual information maximization can be formulated as follows

$$
\max _{x_{k, n}, p_{k, n}} \sum_{k \in \mathcal{K}} w_{k} \sum_{n \in \mathcal{N}} x_{k, n} I\left(\frac{p_{k, n} g_{k, n}}{x_{k, n}}\right),
$$


subject to

$$
\begin{gathered}
\sum_{n \in \mathcal{N}} p_{k, n} \leq P_{k}, \quad p_{k, n} \geq 0, \quad \forall k \in \mathcal{K}, \quad n \in \mathcal{N}, \\
\sum_{k \in \mathcal{K}} x_{k, n} \leq 1, \quad \forall n \in \mathcal{N}, \\
x_{k, n} \in\{0,1\}, \quad \forall k \in \mathcal{K}, \quad n \in \mathcal{N},
\end{gathered}
$$

where $g_{k, n}=\left|h_{k, n}\right|^{2} / N_{0} B_{N}$ is the SNR for user $k$ on subcarrier $n$ with unit power, and $h_{k, n}$ is the channel gain that user $k$ experiences on subcarrier $n . N_{0}$ and $B_{N}$ are the noise power spectral density and the subchannel bandwidth, respectively. $x_{k, n}$ is the subcarrier allocation binary indicator, where $x_{k, n}$ equals to 1 if subcarrier $n$ is allocated to user $k$, and 0 otherwise. $I($.$) denotes the mutual information between$ the input and the output of the channel, and $w_{k}$ is the weight associated with user $k$. As we can see, the power constraint (2) couples the problem among the subcarriers, where the power allocated over the subcarriers has to satisfy the power constraint (2). On the other hand, the subcarrier allocation constraint (3) couples the problem among the users.

\section{Optimal Solution}

The optimization problem (1-4) above is a combinatorial one due to the binary variable $x_{k, n}$, which makes the problem intractable for large system. One approach for solving the problem is to relax the binary constraint and allows any real value in the interval $[0,1]$. Hence, the constraint (4) can be replaced by

$$
x_{k, n} \geq 0, \quad \forall k \in \mathcal{K}, \quad n \in \mathcal{N} .
$$

If the subcarrier allocation is carried out over blocks of OFDMA symbols, the non-integer values of $x_{k, n}$ can be realised by sharing the symbols in the blocks over time. Also, if the number of subcarrier is large enough such that the adjacent subcarriers are highly correlated, then the noninteger values of $x_{k, n}$ can be realised by frequency sharing. It can be easily verified that the relaxed problem is convex and has no duality gap. Consequently, the problem can be solved via decomposition methods [5]. We will solve the relaxed problem by decomposing it into two subproblems; one for power allocation and the other for subcarrier allocation.

First subproblem: for a given subcarrier allocation $\left\{x_{k, n}\right\}$ the power allocation problem will be decomposed into $K$ power allocation subproblems, each one for a single-user

$$
\max _{p_{k, n}} \sum_{n \in \mathcal{N}} x_{k, n} I\left(\frac{p_{k, n} g_{k, n}}{x_{k, n}}\right),
$$

subject to the constraints (2). This subproblem, Single-User Power Allocation (SUPA), is convex and the optimal power allocation can be found as follows.

Corollary 1. The optimal power allocation for (6) is given by

$$
p_{k, n}^{\star}= \begin{cases}\frac{x_{k, n}}{g_{k, n}} \text { mmse }^{-1}\left(\frac{\lambda_{k}}{g_{k, n}}\right) & \text { if } g_{k, n}>\lambda_{k}, \\ 0 & \text { if } g_{k, n} \leq \lambda_{k},\end{cases}
$$

where $\lambda_{k}$ is the Lagrange multiplier associated with the power constraint (2) and can be found using iterative methods (such as bisection, secant, or Newton) by solving the following equation

$$
\sum_{n \in \mathcal{N}}^{\left(g_{k, n}>\lambda_{k}\right)} \frac{x_{k, n}}{g_{k, n}} m m s e^{-1}\left(\frac{\lambda_{k}}{g_{k, n}}\right)-P_{k}=0 .
$$

Proof: See Appendix A.

It is worth mentioning that without the subcarrier allocation index, the optimal SUPA will turn into Mercury/Waterfilling [3].

Second subproblem: for a given power allocation $\left\{p_{k, n}\right\}$ the subcarrier allocation problem will be decoupled into $N$ optimization subproblems, each over one subcarrier

$$
\max _{x_{k, n}} \sum_{k \in \mathcal{K}} w_{k} x_{k, n} I\left(\frac{p_{k, n} g_{k, n}}{x_{k, n}}\right),
$$

subject to the constraints (3) and (5). The optimal subcarrier allocation can by found iteratively using gradient method as follows

$$
x_{k, n}(j+1)=x_{k, n}(j)+\alpha(j) s_{k, n},
$$

where $\alpha(j)$ is a positive step-size, and $s_{k, n}$ is the gradient of (9) with respect to $x_{k, n}$ and given by

$$
s_{k, n}=w_{k} I\left(\frac{p_{k, n} g_{k, n}}{x_{k, n}}\right)-\frac{w_{k} p_{k, n} g_{k, n}}{x_{k, n}} \operatorname{mmse}\left(\frac{p_{k, n} g_{k, n}}{x_{k, n}}\right) .
$$

To satisfy the constraint (3), the updated subcarrier allocation must be normalized as follows

$$
x_{k, n}=\frac{x_{k, n}}{\sum_{k \in \mathcal{K}} x_{k, n}} .
$$

Consequently, the optimal solution of the relaxed problem can be found iteratively by solving the subproblems (6) and (9). Starting with arbitrary feasible subcarrier allocation, the optimal power allocation is found using (7). Using the optimal power allocation, the optimal subcarrier allocation is found using the gradient method. Then, this subcarrier allocation is fed back into the power allocation problem, and so on. After large number of iterations, the algorithm will reach the optimal solution of the relaxed problem. The proposed iterative algorithm is detailed in Algorithm 1. As evidenced by (10) and (11), the optimal subcarrier allocation will allocate more subcarrier share to the user who has the highest gradient (11) on that subcarrier. Consequently, the objective function will be increased. As the objective function is bounded due to the limited power and subcarriers, the increase in the objective function continues until it reaches to an equilibrium point, which is the global optimal as the optimization problem is a convex one. For the termination criterion, the iterative algorithm terminates if either the number of iterations reaches a given threshold or the difference in the objective function between two successive iterations vanishes.

\section{Suboptimal Algorithm}

The optimal SPA is impractical for real time implementation due to the following reasons. Firstly, the complexity for solving the relaxed problem is too high for practical 


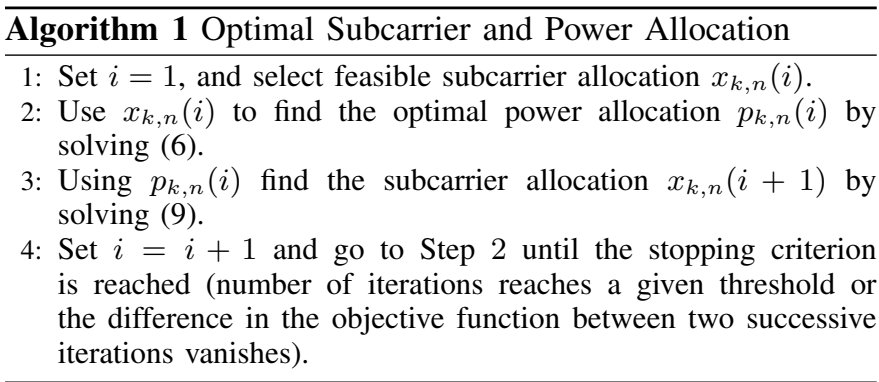

systems. Secondly, partitioning of the available bandwidth in optimal fashion may be difficult to achieve in practice. Furthermore, although optimal power allocation maximizes the user mutual information, signalling the power levels to the users requires high signalling overhead, which will reduce the spectral efficiency. Thus, it is more practical to use equal power allocation over the allocated subcarriers to the user [6]. In this way, only the subcarrier allocation decisions need to be signalled to the users. Consequently, in this section, we propose a suboptimal algorithm that takes into account more practical assumptions. The algorithm relies on equal power allocation that is performed on appropriately selected set of subcarriers. Each user will allocate its power only on the set of the subcarriers that have non-zero power in the optimal SUPA. Let $N_{k}^{\star}$ be the number of subcarriers that will have non-zero power under optimal SUPA, the equal power allocation can be expressed as

$$
p_{k, n}= \begin{cases}\frac{P_{k}}{N_{k}^{\star}} & \text { if } g_{k, n}>\lambda_{k}, \\ 0 & \text { if } g_{k, n} \leq \lambda_{k}\end{cases}
$$

where $\lambda_{k}$ is the solution of (8). It is clear that the optimal Lagrange multiplier $\lambda_{k}$ is required to perform the power allocation (13). Using the numerical methods (such as bisection or Newton) to find $\lambda_{k}$ by solving (8) is computationally inefficient due to the lack of closed-form expressions of the $\mathrm{mmse}($.$) and \mathrm{mmse}^{-1}($.$) functions. Here, we propose a$ simplified iterative method to calculate the optimal Lagrange multiplier based on an approximation of the mmse(.) function.

Lemma 1. For a given range of $S N R, m_{m s} e^{-1}($.$) can be$ approximated by

$$
m m s e^{-1}(x)=\frac{\log _{10}(x)-B}{A},
$$

where the constants $A$ and $B$ are defined in Appendix $B$.

Proof: See Appendix B.

Using (14) and (8), for a given set of subcarriers, $\lambda_{k}$ can be calculated using the following equation

$$
\log _{10}\left(\lambda_{k}\right)=\frac{P_{k}+\sum_{n \in \mathcal{T}_{k}}\left(\frac{\log _{10}\left(g_{k, n}\right)+B}{g_{k, n} A}\right)}{\sum_{n \in \mathcal{T}_{k}} \frac{1}{g_{k, n} A}} .
$$

Consequently, the optimal Lagrange multiplier can be iteratively found, by sorting the subcarriers in a descending order based on their channel gains. Then, starting with the best

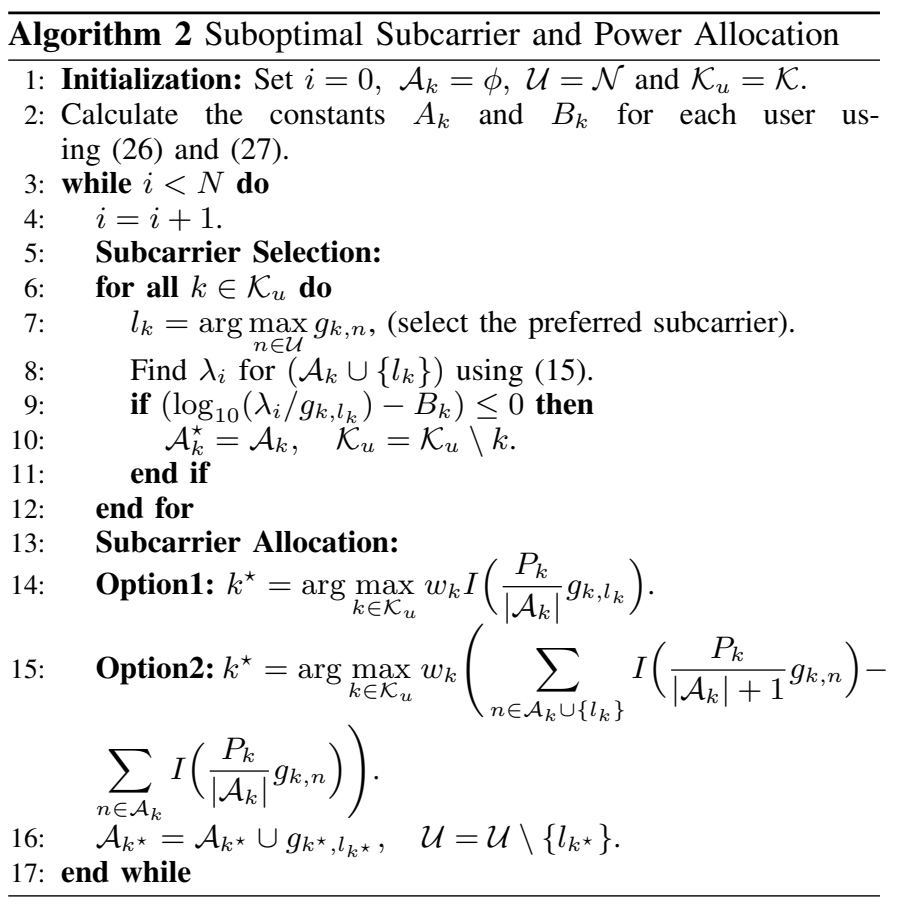

TABLE I

ALGORITHM COMPLEXITY

\begin{tabular}{|l||c|c|c|c|}
\hline \multicolumn{1}{|c||}{} & \multicolumn{4}{c|}{ Operation } \\
\cline { 2 - 5 } & $\max ()$. & $\log ($.$) / Exp.$ & Add./Subt. & Multip./Div. \\
\hline Option1 & $K N$ & $N(3 K+N / 2)$ & $N(4 K+N)$ & $N(7 K+N)$ \\
\hline Option2 & $K N$ & $N(4 K+3 N / 2)$ & $2 N(3 K+N)$ & $2 N(4 K+N)$ \\
\hline
\end{tabular}

subcarrier, $\lambda_{k}$ is calculated using (15). Another subcarrier is added, with a descending order, in each step as long as it results in positive power allocation, until a non-positive power allocation occurs.

Using the proposed approximation and equal power allocation, the suboptimal SPA can be developed in two phases. In the first phase (Subcarriers Selection), each user select its best unallocated subcarrier, referred to as the preferred subcarrier. Also, a check is performed to see if the user has reached a saturation point, i.e., the preferred subcarrier will have zero power under optimal SUPA. In the second phase (Subcarrier Allocation), a subcarrier is allocated to one user based on the users' utilities with equal power allocation. We will consider two options for subcarrier allocation criteria. The first option is to consider the utility as the user's weighted mutual information on its preferred subcarrier. For the second option, the utility is considered as the increase in the user's mutual information due to allocating an extra subcarrier. The algorithm iteratively allocates a subcarrier in each iteration until all the subcarriers are allocated. The proposed suboptimal algorithm is explained in details in Algorithm 2. Different mathematical operations necessary for the implementation of our algorithm are identified. We count the instances of each of these operations in each step of the algorithm and multiply it by the number of times this step is performed in a complete run of the algorithm. These total counts for each type of operation are provided in Table I. It can be seen from the table that both Option1 and Option2 have a complexity order of $\mathcal{O}(K N)$. 


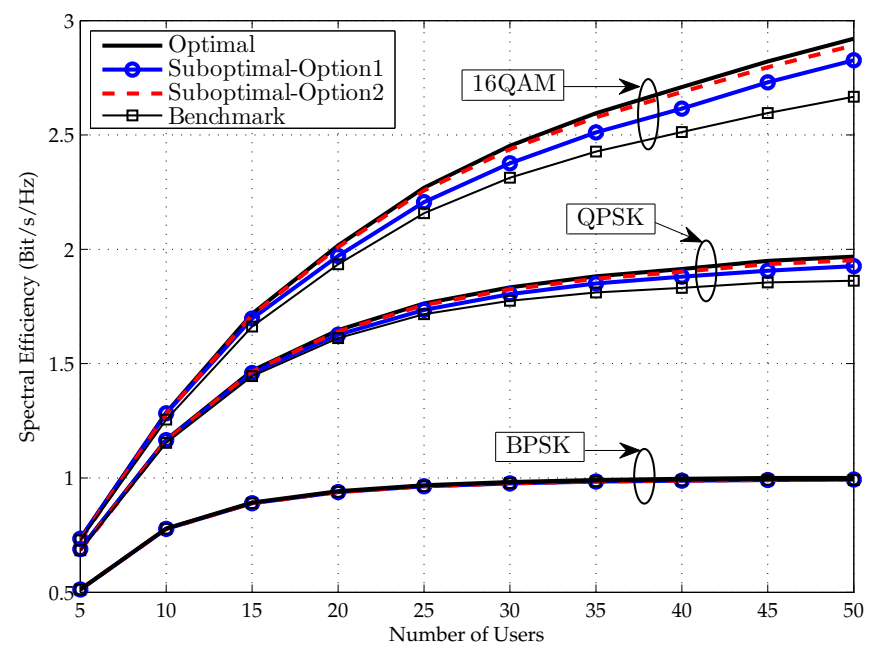

Fig. 1. Spectral efficiency comparison of the proposed algorithm and the optimal solution.

Comparing to the optimal solution and the benchmark algorithm that have complexity order of $\mathcal{O}\left(L K N\left(L_{1}+L_{2}\right)\right)$ and $\mathcal{O}\left(K N^{2}\right)$, respectively, our algorithm has much lower complexity. Here, $L_{1}$ is the number of iterations required to find the optimal power (8) in the First Subproblem, $L_{2}$ is the number of iterations for the gradient method (10-12) in the Second Subproblem, and $L$ is the number of main iterations in Algorithm 1.

\section{Simulation Results}

In this section, we evaluate the performance of the proposed algorithm through Monte Carlo simulation. A singlecell with $1 \mathrm{~km}$ radius will be considered, and users' locations are randomly generated and uniformly distributed over the cell. The total bandwidth is $5 \mathrm{MHz}$ consisting of 32 subcarriers and the user's maximum transmit power is 1 Watt. ITU pedestrian B is adopted for fast fading generation [7, page 476], and the simplified model [8] for the path loss. The noise power spectral density is assumed to be $-120 \mathrm{~dB} / \mathrm{Hz}$. The performance is compared with the optimal solution of the relaxed problem and a benchmark algorithm from [9], which has been proven to be Pareto optimal within a large neighbourhood of the solution obtained by the algorithm [9]. In the simulations, the optimal algorithm stops if the gain in spectral efficiency between two successive iterations is less than $10^{-3} \mathrm{bit} / \mathrm{s} / \mathrm{Hz}$, and the maximum number of iterations was 200. The algorithm required about $60-150$ iterations on average to converge, depending on the number of users $(K)$. Fig. 1 shows the spectral efficiency versus the number of users $(K)$ for different modulation orders (BPSK, QPSK and 16QAM). It can be seen that the performance of our proposed suboptimal algorithm, with the two subcarrier allocation criteria (Option1 and Option2), achieves very close performance to the upper bound and outperforms the benchmark algorithm. The proposed algorithm achieves in average $98.2 \%$ and $99.4 \%$ of the optimal solution for Option 1 and Option2, respectively. Furthermore, the gap between the two subcarrier allocation criteria (Option1 and Option2) increases as the modulation order

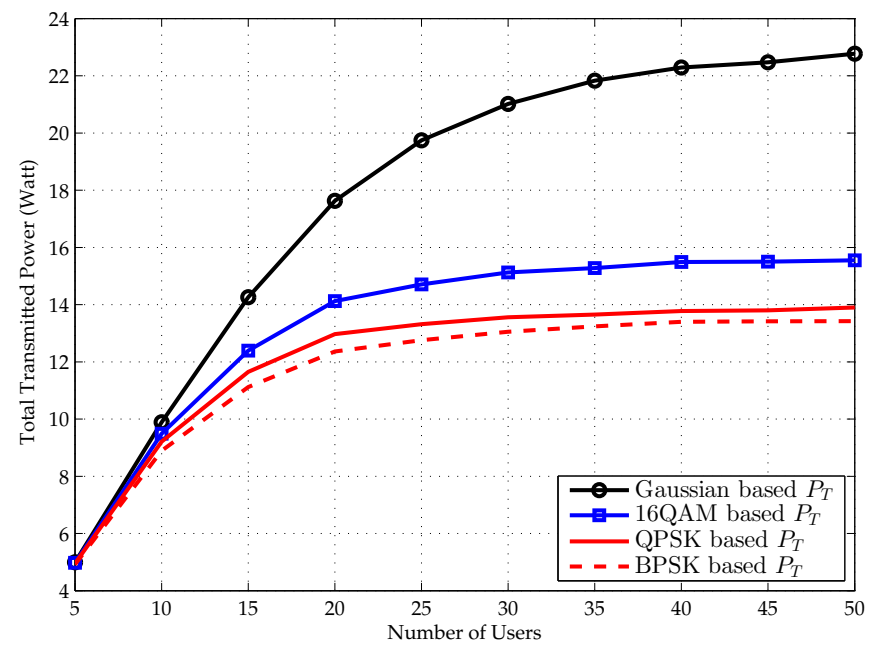

Fig. 2. Comparison of the total transmitted power, $P_{T}$, for FSA and Gaussian inputs.

increase, and Option2 always outperforms Option1. Now, the advantage of explicitly considering the FSA in optimizing the power allocation comparing to the Gaussian assumption will be demonstrated. Fig. 2 shows the total transmitted power for each channel input (Gaussian and FSA) versus the total number of users in the system. The total transmitted power, $P_{T}$, is given by

$$
P_{T}=\sum_{k \in \mathcal{K}} \sum_{n \in \mathcal{N}} p_{k, n} .
$$

As the figure shows, the total transmitted power under Gaussian assumption is higher comparing to the FSA inputs. If the power is optimized based on the Gaussian input then FSA is used for transmission, large amount of power will be wasted. Consequently, using the proposed algorithm will significantly reduce the average transmitted power of each user. Thus, as the achieved spectral efficiency is the same for both cases, our algorithm can be considered more energy efficient comparing to the algorithms that assumes Gaussian inputs. To evaluate the effect of imperfect CSI on the algorithm performance, we expressed the imperfect CSI as $\hat{g}_{k, n}=g_{k, n}+e_{k, n}$, where $g_{k, n}$ is the perfect CSI and $e_{k, n}$ is the error in the CSI estimation, which modeled as a zero-mean Gaussian random variable with variance $\sigma_{e}^{2}$ [10]. Fig. 3 shows the spectral efficiency of the proposed algorithm for different values of error variance $\left(\sigma_{e}^{2}\right)$. As can be observed from the figure, the effect of imperfect CSI on the algorithm's performance is marginal. For the simulated error variance, the maximum spectral efficiency degradation are $0.14 \%$ and $0.09 \%$ for Option 1 and Option2, respectively, which can be considered practically negligible.

\section{Conclusion}

In this paper, we considered radio resource allocation for weighted sum mutual information maximization in the uplink OFDMA system. Unlike existing work that assumes Gaussian inputs, we consider FSA inputs. By capitalizing on the relationship between mutual information and the MMSE, we provided the optimal solution using a decomposition approach. 


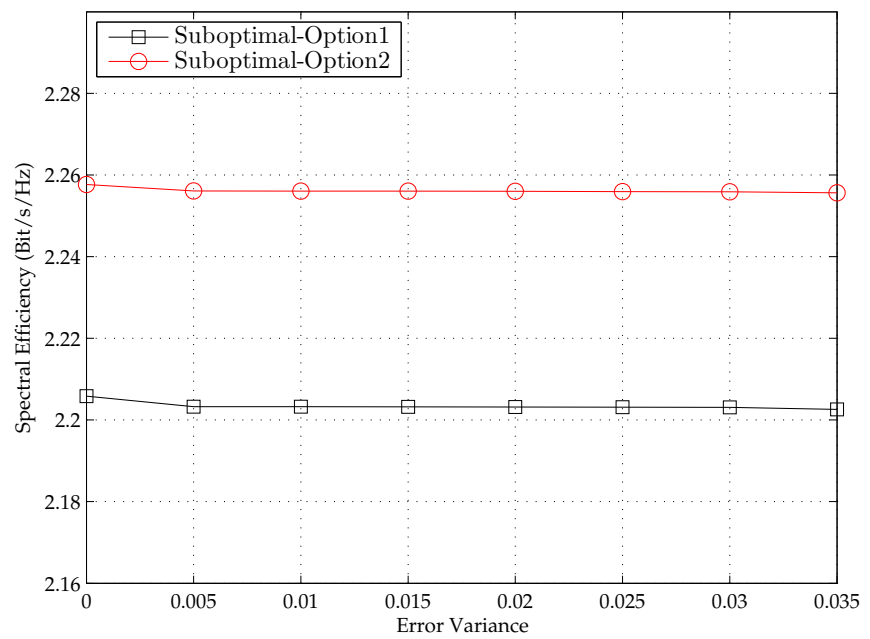

Fig. 3. Effect of imperfect CSI on the performance of proposed suboptimal algorithm (16QAM, 25 users).

Due to the complexity of the optimal solution, we proposed a low-complexity suboptimal subcarrier and power allocation algorithm. The complexity of the proposed algorithm is analysed and shown to have complexity of $\mathcal{O}(K N)$, which is far less than that of the optimal solution and the benchmark algorithm. The performance of the proposed algorithm is evaluated via Monte Carlo simulation and shown to achieve near optimal performance and outperform the benchmark algorithm. Furthermore, it is revealed that by explicitly optimizing the power for FSA inputs, significant power saving can be gained comparing to the case when Gaussian input is assumed.

\section{ACKNOWLEDGEMENT}

This study was sponsored by the UK Engineering and Physical Sciences Research Council (EPSRC) under grant number EP/J017655/1. The authors would like to acknowledge its financial support.

\section{APPENDIX A}

\section{PROOF OF COROLlARY 1}

Proof: The subproblem (6) is convex and it satisfies the Slater condition (by setting $p_{k, n}=P_{k} / N$ ). Hence, there is no duality gap and the Karush-Kuhn-Tucker (KKT) conditions are necessary and sufficient. The Lagrangian for subproblem (6) can be formulated as

$$
\begin{aligned}
& L_{k}\left(\mathbf{p}_{k}, \lambda_{k}, \boldsymbol{\nu}_{k}\right)=-\sum_{n \in \mathcal{N}} x_{k, n} I\left(\frac{p_{k, n} g_{k, n}}{x_{k, n}}\right)+ \\
& \lambda_{k}\left(\sum_{n \in \mathcal{N}} p_{k, n}-P_{k}\right)-\sum_{n \in \mathcal{N}} \nu_{k, n} p_{k, n},
\end{aligned}
$$

and the KKT conditions are

$$
\begin{gathered}
-g_{k, n} \operatorname{mmse}\left(\frac{p_{k, n}^{\star} g_{k, n}}{x_{k, n}}\right)+\lambda_{k}-\nu_{k, n}=0, \\
\nu_{k, n} \geq 0, \quad p_{k, n}^{\star} \geq 0, \quad \forall n \in \mathcal{N}, \\
\nu_{k, n} p_{k, n}^{\star}=0, \quad \forall n \in \mathcal{N} .
\end{gathered}
$$
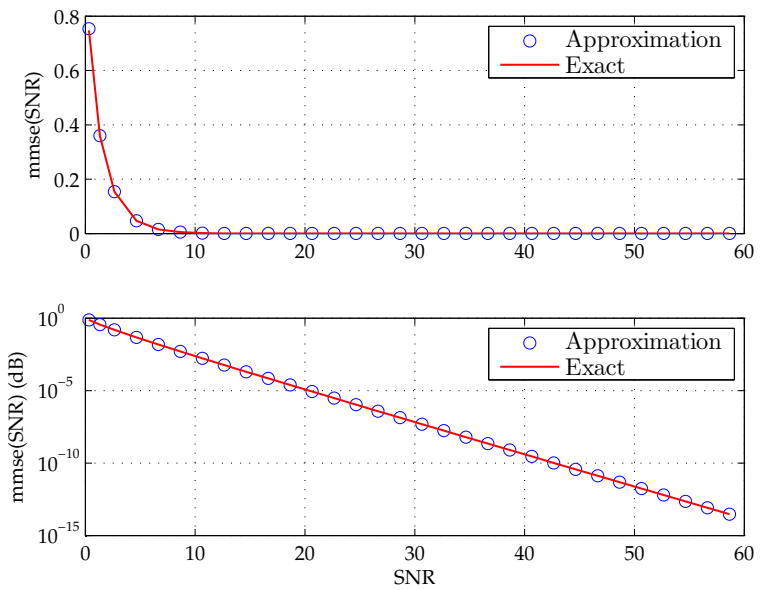

Fig. 4. Linear approximation for the MMSE (the QPSK case).

Using (18) and (20), and by substituting (18) into (19) yields

$$
\begin{gathered}
p_{k, n}^{\star}\left(\lambda_{k}-g_{k, n} \operatorname{mmse}\left(\frac{p_{k, n}^{\star} g_{k, n}}{x_{k, n}}\right)\right)=0, \\
g_{k, n} \operatorname{mmse}\left(\frac{p_{k, n}^{\star} g_{k, n}}{x_{k, n}}\right) \leq \lambda_{k} .
\end{gathered}
$$

Consequently, if $p_{k, n}^{\star}>0$ then from (21) we have $\lambda_{k}=$ $g_{k, n} \operatorname{mmse}\left(\frac{p_{k, n}^{\star} g_{k, n}}{x_{k, n}}\right)$, therefore

$$
p_{k, n}^{\star}=\frac{x_{k, n}}{g_{k, n}} \mathrm{mmse}^{-1}\left(\frac{\lambda_{k}}{g_{k, n}}\right) .
$$

Since mmse $\left(\frac{p_{k, n}^{\star} g_{k, n}}{x_{k, n}}\right)<1$ when $p_{k, n}^{\star}>0$, we know from (22) that $g_{k, n}>\lambda_{k}$. On the other hand, as the $\operatorname{mmse}(0)=1$, if $p_{k, n}^{\star}=0$, we have from (22) $g_{k, n} \leq \lambda_{k}$. Hence, the corollary follows.

\section{APPENDIX B}

\section{MMSE APPROXIMATION (proof of Lemma 1)}

For FSA sets, there is no closed-form expression for the MMSE, and it is calculated numerically using the following formula

$$
m m s e(s n r)=1-\frac{1}{\pi} \int \frac{\left|\sum_{x \in \mathcal{X}} p(x) x e^{-|y-\sqrt{s n r} x|^{2}}\right|^{2}}{\sum_{x \in \mathcal{X}} p(x) e^{-|y-\sqrt{s n r} x|^{2}}} d y,
$$

where the integral is over the complex field and $p(x)$ is the probability of $x$ [3]. It is clear that using the numerical methods to calculate the MMSE from this formula to find the optimal Lagrange multiplier (8) is computationally complex. As can be seen in Fig. 4, the logarithm of the mmse(.) is almost linear in the SNR. Consequently, a linear approximation of the logarithm of the mmse(.) for a given range of the SNR can be formulated as follows

$$
\begin{aligned}
\log _{10}(m m s e(s n r))= & A s n r+B, \\
& S N R_{\text {min }} \leq s n r \leq S N R_{\text {max }},
\end{aligned}
$$


where $A$ is the slop of the line, and $B$ is the y-intercept, which are given by

$$
A=\frac{\log _{10}\left(m m s e\left(S N R_{\max }\right)\right)-\log _{10}\left(\operatorname{mmse}\left(S N R_{\min }\right)\right)}{S N R_{\max }-S N R_{\min }},
$$

$$
B=\log _{10}\left(m m s e\left(S N R_{m i n}\right)\right)-A S N R_{m i n} .
$$

The error in calculating the MMSE using the linear approximation is considerably low. For the results presented in Fig. 4, the mean squared error $\mathbb{E}(\operatorname{mmse}(s n r)-\widehat{\operatorname{mmse}}(s n r))^{2}$ is only $7 \times 10^{-7}$ for the QPSK case, and the SNR range is $30 \mathrm{~dB}$. Similar results have been noticed for other modulation orders. The $\mathrm{mmse}^{-1}($.$) can be straightforwardly found from (25) as$ follows

$$
\operatorname{mmse}^{-1}(x)=\frac{\log _{10}(x)-B}{A} .
$$

\section{REFERENCES}

[1] E. Yaacoub and Z. Dawy, "A survey on uplink resource allocation in OFDMA wireless networks," IEEE Commun. Surveys Tuts., vol. 14, no. 2, pp. 322-337, Second Quarter 2012.

[2] B. Devillers, J. Louveaux, and L. Vandendorpe, "Bit and power allocation for goodput optimization in coded parallel subchannels with ARQ," IEEE Trans. Signal Process., vol. 56, no. 8, pp. 3652 -3661, Aug. 2008.

[3] A. Lozano, A. Tulino, and S. Verdu, "Optimum power allocation for parallel Gaussian channels with arbitrary input distributions," IEEE Trans. Inf. Theory, vol. 52, no. 7, pp. 3033-3051, July 2006.

[4] D. Guo, S. Shamai, and S. Verdú, "Mutual information and minimum mean-square error in Gaussian channels," IEEE Trans. Inf. Theory, vol. 51, no. 4, pp. 1261-1282, Apr. 2005.

[5] D. Palomar and M. Chiang, "A tutorial on decomposition methods for network utility maximization," IEEE J. Sel. Areas Commun., vol. 24, no. 8, pp. 1439-1451, Aug. 2006.

[6] W. Yu and J. Cioffi, "Constant-power waterfilling: performance bound and low-complexity implementation," IEEE Trans. Commun., vol. 54, no. 1, pp. 23-28, Jan. 2006.

[7] L. Korowajczuk, LTE, WiMAX and WLAN Network Design, Optimization and Performance Analysis. John Wiley \& Sons Ltd, 2011.

[8] A. Goldsmith, Wireless Communications. Cambridge University Press, 2005.

[9] C. Y. Ng and C. W. Sung, "Low complexity subcarrier and power allocation for utility maximization in uplink OFDMA systems," IEEE Trans. Wireless Commun., vol. 7, no. 5, pp. 1667-1675, May 2008.

[10] P. Xiao and E. Ström, "Soft demodulation algorithms for orthogonally modulated and convolutionally coded DS-CDMA systems," IEEE Trans. Commun., vol. 58, no. 3, pp. 742-747, Mar. 2010. 